\title{
Zur Riskanz von Veränderungsprozessen: Faktoren ihres Ge- oder Misslingens
}

\section{Astrid Schreyögg}

(C) Springer Fachmedien Wiesbaden 2016

Alle Welt redet von Change. Veränderung ist allgegenwärtig. Ist sie aber überall möglich? Ist sie überall erfolgreich? Womöglich ist sie hier und da fast undenkbar. Bei genauerer Betrachtung ist jede Veränderung nicht nur für die Change Agents, sondern auch für diejenigen, die verändert werden sollen, seien es Menschen oder ganze Organisationen, riskant. Veränderung kann gelingen, kann aber auch misslingen. Einzelne Menschen und soziale Systeme sind nämlich keine Gegenstände, die beliebig manipulierbar sind, sondern Subjekte, die sich jedem Veränderungsversuch entziehen oder diesen sogar konterkarieren können. Das sollte sich jeder Change Agent, trotz aller vermeintlichen oder tatsächlichen Erfolge, immer wieder deutlich machen. So befassen wir uns im vorliegenden Heft mit der generellen Riskanz von Veränderungsprozessen, d.h. mit der Art, wie sie gelingen oder wie sie umgekehrt auch misslingen können.

Regina Heimann liefert uns zunächst einen übergeordneten, gleichsam einen Meta-Rahmen für das Verständnis von Change-Prozessen. Sie zeigt auf dem Hintergrund des Habitus-Konzepts von Bourdieu, wie umfassend die jeweilige Perspektivität von Beratern und Ratsuchenden den Erfolg versus Misserfolg von Beratung bestimmt. Dabei geht es der Autorin besonders um sozial determinierte Konfliktdimensionen mit ihren Entwicklungsbarrieren.

Im zweiten Beitrag von Florian Zapp werden „Ambivalenzen und Widersprüche“ in Workshops thematisiert. Der Autor spricht hier nicht nur quasi vor- oder gar unbewusste Widersprüche an, sondern auch rationale Vorbehalte. Zu diesem Zweck stellt er ein Konzept vor, mit dessen Hilfe die „Widerspruchstoleranz“ verbessert werden soll.

Dr. A. Schreyögg ( $\triangle)$

Breisgauer Str. 29, 14129 Berlin, Deutschland

E-Mail: info@schreyoegg.de 
Michaela Riediger befasst sich mit einem sehr ernsten Problem, an dem sich viele Coaches die Zähne ausbeißen, nämlich mit Prokrastination von Klienten. Dabei handelt es sich um psychogenes Aufschieben, also um solches, das weder vom Betreffenden selbst noch von der Umgebung intendiert ist. Solche „Aufschieberitis“ ist nach Meinung der Autorin allerdings mit Hilfe der „Temporal Motivation Theory“ $\mathrm{zu}$ erfassen und zu bearbeiten.

Umgekehrt mag Selbstmanagement von Führungskräften, wie Jörg Pscherer in einer Online-Studie untersucht hat, solch unguten und zähen Prozessen vorbeugen. Im Gegenteil, gute Selbstregulierung zieht, wie der Autor meint, gute Resilienz und dann auch Arbeitserfolg nach sich.

Günther Mohr erweitert den Rahmen von Resilienz und schreibt über ,,systemische Resilienz". Der Autor hat untersucht, wie sich das Konzept individueller Widerstandskraft auf organisatorische Systeme übertragen lässt. Er hatte dabei besonders Resilienz-Faktoren bei Change-Prozessen im Blick. Seiner Meinung nach gibt es eine Entsprechung zwischen individuellen und systemischen Resilienz-Phänomenen.

Ingo Zimmermann präsentiert eine grundlegende Kritik an der Sicht von Barrieren in Veränderungsprozessen. Dieser Autor meint, dass bei den meisten Coaches, aber auch Coaching-Autoren perspektivisch die Methodenebene dominiert. Metatheoretische Überlegungen stellten die Ausnahme dar. Zur Abhilfe bietet er das Konzept des „Capability Approach“, das im Anschluss an Theorien von Johan Galtung entwickelt wurde.

Eric de Haan betont, dass die Qualität von Organisationen besonders durch Supervision und Coaching gefördert werden könne. Durch diese Formate sei am ehesten die Balance zwischen Stabilität und Veränderung zu garantieren.

Im Praxisbericht thematisiert Isabell Diermann das Image von Supervision in Kliniken. Dabei erweist es sich, dass das Image keineswegs optimal ist. Und im Diskurs plädiert Thomas Reyer für mehr Klarheit und Abgrenzung zwischen den verschiedenen Beratungsformaten. 